\title{
The efficacy of a sumatriptan and naproxen combination pill in a patient with chronic migraines who discontinued triptan therapy in the past due to a self-reported poor response to sumatriptan monotherapy
}

Ethan M. Cohen

Wayne State University School of Medicine, ethan.cohen2@med.wayne.edu

Follow this and additional works at: https://digitalcommons.wayne.edu/crp

Part of the Medical Education Commons, Neurology Commons, Primary Care Commons, and the Therapeutics Commons

\section{Recommended Citation}

COHEN EM. The efficacy of a sumatriptan and naproxen combination pill in a patient with chronic migraines who discontinued triptan therapy in the past due to a self-reported poor response to sumatriptan monotherapy. Clin. Res. Prac. Oct 13 2021;7(2):eP2866. https://doi.org/10.22237/crp/ 1625097900

This Clinical Decision Report is brought to you for free and open access by the Open Access Journals at DigitalCommons@WayneState. It has been accepted for inclusion in Clinical Research in Practice: The Journal of Team Hippocrates by an authorized editor of DigitalCommons@WayneState. 
The efficacy of a sumatriptan and naproxen combination pill in a patient with chronic migraines who discontinued triptan therapy in the past due to a selfreported poor response to sumatriptan monotherapy

Cover Page Footnote 


\title{
The efficacy of a sumatriptan and naproxen combination pill in a patient with chronic migraines who discontinued triptan therapy in the past due to a self- reported poor response to sumatriptan monotherapy
}

ETHAN M. COHEN, BS, Wayne State University School of Medicine, ethan.cohen2@med.wayne.edu

\author{
ABSTRACT A clinical decision report using:
}

Mathew NT, Landy S, Stark S, et al. Fixed-dose sumatriptan and naproxen in poor responders to triptans with a short half-life. Headache. 2009;49(7):971-982. https://doi.org/10.1111/j.1526-4610.2009.01458.x

for a patient with chronic migraines who had self-reported poor response to sumatriptan monotherapy.

Keywords: $\quad$ sumatriptan, naproxen, migraine, monotherapy, chronic, lifestyle, NSAID

\section{Clinical-Social Context}

Ms. Sue Brown [pseudonym] is a 36-year-old African American female that presented to the medicine clinic to discuss her persistent migraine symptoms despite prior attempts at pharmacological intervention. Her past medical history is significant for hypertension, opioid use disorder, and migraines. Her hypertension has been well controlled for three years on lisinopril 20mg and chlorthalidone $50 \mathrm{mg}$. Ms. Brown's surgical history includes a cesarean section six years ago. After the procedure, Ms. Brown was prescribed morphine for pain control, to which she subsequently developed dependence. After a year of opioid dependence, she attended rehabilitation and has been abstinent. She currently lives in a suburban neighborhood with her two children and boyfriend and feels safe at home. She works as a cashier, is enrolled in Medicaid, and is able to support her family's needs with the addition of her boyfriend's salary. She noted that she has adequate access to healthcare and food, is using the Nexplanon implant for contraception, and has family close by that helps her take care of her children when she works. She has a 10th grade education and does not smoke tobacco, drink alcohol, or use any drugs.

Ms. Brown noted that she has had right-sided migraines lasting 8-14 hours for years without a clear trigger. During her migraines, she is unable to leave her bed as light and sound worsen her symptoms. Initially, her migraines were intermittently controlled on oral sumatriptan $100 \mathrm{mg}$. Although not optimal, Ms. Brown noted that this was the most effective solution so far, so she stuck with it to see if it would improve over time. Due to the loss of a family member and the stress of the COVID-19 pandemic, her migraines no longer respond to medication and occur 6-8

ETHAN M. COHEN, BS, is a student at the Wayne State University School of Medicine. 
COHEN EM. The efficacy of a sumatriptan and naproxen combination pill in a patient with chronic migraines who discontinued triptan therapy in the past due to a self-reported poor response to sumatriptan monotherapy. Clin. Res. Prac. Oct 13 2021;7(2):eP2866.

https://doi.org/10.22237/crp/1625097900
Clinical Research in Practice The Journal of Team Hippocrates

VOL 7 ISS 2 / eP2866 / OCTOBER 13, 2021 https://doi.org/10.22237/crp/1625097900

times per month. During this time, Ms. Brown discontinued her use of sumatriptan as it was no longer effective. She now misses work and family time due to her symptoms. She wishes to restart sumatriptan with an additional medication because she had not had any prior side effects. Additionally, she has a family member with similar migraines that was treated with naproxen, so she is interested in seeing if that could help.

\section{Clinical Question}

Does the addition of naproxen to a triptan significantly reduce severe migraine symptoms for a patient that has already taken and discontinued sumatriptan due to decreasing efficacy in treating recurrent migraines?

\section{Research Article}

Mathew NT, Landy S, Stark S, et al. Fixed-dose sumatriptan and naproxen in poor responders to triptans with a short half-life. Headache. 2009;49(7):971-982. https://doi.org/10.1111/i.1526-4610.2009.01458.x ${ }^{1}$

\section{Description of Related Literature}

The literature search was performed using PubMed advanced key word searches. The search began by selecting for clinical trials (CT) and randomized control trials (RCT) with the key terms "migraine" and "sumatriptan." This search revealed 396 manuscripts, so the search criteria needed to be narrowed.

The next search selected for CT and RCT after the year 2005, using the key terms "migraine," "sumatriptan," and "naproxen." This yielded 30 results, of which three discussed menstruation-related migraines, three used adolescents as their study group, and four discussed the pharmacokinetics of medications, so these could be excluded from further review. In addition, Ms. Brown had been taking sumatriptan for a number of years, so 17 of the remaining 20 manuscripts could be excluded because the patients were either triptan naïve or prescribed a different abortant migraine medication prior to the study. This left three studies which are reviewed below.

Landy et al., 2007 is a RCT that looked at patients self-reported ability to function, be productive, and satisfied after taking a combined pill of sumatriptan $85 \mathrm{mg}$ and naproxen $500 \mathrm{mg}$ compared to a control. Patients were more satisfied, productive, and functional with the combined medication than the other treatment groups. $\underline{2}$ The study cohort did not include groups that had poor response to triptans, so it cannot be expanded to include Ms. Brown.

Edwards et al., 2012 is a RCT that assessed patient's cognitive function before and after treatment for migraine as an indicator of migraine severity using either a combination drug of sumatriptan $85 \mathrm{mg}$ and naproxen $500 \mathrm{mg}$ or a control. The authors found improvement in recovery time and cognitive ability after treatment with the combined medication. $\underline{3}$ Unlike Ms. Brown, these patients were well-controlled on their migraine medications prior to enrollment in the study.

Mathew et al., 2009 is a RCT that evaluates the efficacy and tolerability of the combined sumatriptan $85 \mathrm{mg}$ and naproxen $500 \mathrm{mg}$ medication against a control in patients with severe migraines that were unable to continue triptan treatment alone due to poor response. The authors identified benefit of this medication compared to the placebo in all endpoints, indicating efficacy of the drug in this cohort. 1

This manuscript was chosen as the most appropriate to answer our clinical question of the efficacy of sumatriptan/naproxen in a cohort with a history of limited efficacy with sumatriptan alone. While it was not the only manuscript to identify the efficacy of the sumatriptan/naproxen combined medication, it was the only one to do so in a group that had poor responsiveness to sumatriptans in the past. $-\underline{5}$ Thus, it has clinical significance in this cohort. Notably, the significant benefit found in this study with the sumatriptan/naproxen combined medication was also seen in other studies that used patients that were triptan-naïve and patients that were effectively treated with sumatriptan before the study. This study can be defined as Level A evidence when referencing the Strength of Recommendations Taxonomy (SORT) criteria due to its high quality as an RCT with support from other published literature. $\underline{6}$ 
COHEN EM. The efficacy of a sumatriptan and naproxen combination pill in a patient with chronic migraines who discontinued triptan therapy in the past due to a self-reported poor response to sumatriptan monotherapy. Clin. Res. Prac. Oct 13 2021;7(2):eP2866.

https://doi.org/10.22237/crp/1625097900
Clinical Research in Practice The Journal of Team Hippocrates

VOL 7 ISS 2 / eP2866 / OCTOBER 13, 2021 https://doi.org/10.22237/crp/1625097900

\section{Critical Appraisal}

The Mathew et al., 2009 manuscript describes two replicate randomized, double-blinded, placebo-controlled, crossover studies. 1 This crossover design allowed the authors to enroll a smaller number of patients while still obtaining the same amount of data as each patient participated as both a placebo and treatment. In a self-reported pain-derived study every patient quantifies their level of pain in a different way. Using patients as both a control and treatment decreased the between-subject variability. The study design allowed for effective randomization because each patient participated in both groups, so the only randomization occurred during the first screening visit before any recording of migraines and consisted of which treatment came first.

It can be argued that these studies meet criteria to be classified as moderate to strong Strength of Recommendation. The studies were blinded, had intention-to-treat analysis, and were of adequate follow-up and size with the crossover design in place. This was the first study to analyze more than one short-acting triptan at a time in poor responders. Thus, the data is not strongly supported with other similar RCTs, decreasing this manuscript's SORT Level of Evidence to $2 . \underline{6}$

These studies recruited patients between the ages of 18-65 with a poorly controlled migraine history on short-acting triptans that discontinued triptans prior to enrollment. The patients themselves were enlisted from 48 different sites around the country, with $>90 \%$ originating from headache or neurology clinics. Thus, there was inherent participation bias as only patients with complaints of poorly treated migraines were likely to join the study as they were the ones being seen at these clinics. Because the authors were able to recruit $>120$ patients that successfully documented two migraine attacks, the study had $>95 \%$ power to detect a $20 \%$ difference between the placebo and treatment. Thus, there were significant similarities between Ms. Brown and the study populations. Additionally, there was an attrition rate of $24 \%$ in one study and $22 \%$ in the second study. This is a notably high attrition rate, especially for a population suffering from frequent migraines that is looking for an effective treatment. As the study mentions, a majority of the patients that did not continue in the study were lost to follow-up or did not have enough migraines to be included. While the patients that were lost to follow up could have had poor response to the treatment so they stopped following the protocol, those that were removed from the study population due to a low number of migraines could have had improvement with treatment. Thus it was not necessarily just the naproxen and triptan treatment regimen that caused the high attrition rate, but the interest of the patients and severity of their disease. That said, follow-up studies with a broader patient population should be conducted to confirm this finding.

The patients selected for the study were very similar to Ms. Brown, but not perfect. While she did not meet any of the exclusion criteria, she was African American. Only 3\% of the cohort in study 1 and $7 \%$ in study 2 were African American, with $>89 \%$ in both studies reporting as white. While unlikely to change the results of the manuscript, it is notable that she fell in the minority patient population studied. In addition, only $9 \%$ and $14 \%$ of patients in study 1 and study 2 were recorded to have had poor response to sumatriptan. Even though she was included into this minority, the results showed an average improvement in every fast-acting triptan included into the study groups, so this is unlikely to have an effect on her response.

To effectively provide data on the efficacy and tolerability in patients that were self-reported non-responders to triptan medication, these two studies attempted to report patient-oriented results. The primary endpoint for efficacy of the combined sumatriptan/naproxen medication was 2-24 hours of a sustained pain-free response. The second endpoint was the percentage of patients without pain 2 hours post dose. Both of these endpoints focus on the patient experience of migraines as their baseline to identify the medication's effectiveness. Both of the efficacy and the tolerability endpoints were reported in the study. The study medication was found to be significantly more effective than placebo for abortion of migraine pain for a 2-24 hour sustained painfree response as well as 2 hours post dose. The percentage of patients with at least one adverse event due to drug-related effects was $8 \%$ and $7 \%$ in the two studies for the study medication. Of these adverse events, only one was deemed more common in the treatment than placebo group, and this adverse effect was also seen in triptan monotherapy, so it was not believed to be caused by the interaction between the combined medication.

The therapeutic maneuver highlighted in the manuscript is feasible in practice, especially in Ms. Brown. The authors instructed patients to take the medication within 1 hour of migraine attack onset, while the pain was still mild. Unlike other studies that waited until the pain was more severe, this increased the functional capacity of the study medication and is easily applicable to a broad population. $\underline{7}, \underline{1}$ 
COHEN EM. The efficacy of a sumatriptan and naproxen combination pill in a patient with chronic migraines who discontinued triptan therapy in the past due to a self-reported poor response to sumatriptan monotherapy. Clin. Res. Prac. Oct 13 2021;7(2):eP2866.

https://doi.org/10.22237/crp/1625097900
Clinical Research in Practice The Journal of Team Hippocrates

VOL 7 ISS 2 / eP2866 / OCTOBER 13, 2021 https://doi.org/10.22237/crp/1625097900

A major confounding factor for this study was the support from GlaxoSmithKline (GSK). Not only did members of the organization support the RCT, but they also had input into the study design, data interpretation, and even participated in critical review of the manuscript. While all of the data collected was presented in the manuscript, the strong participation from GSK presents an increased likelihood of bias as they had a vested interest in the results.

\section{Clinical Application}

Ms. Brown presented to our clinic with poor response to her sumatriptan monotherapy, having 6-8 migraines per month. Her migraines were impacting her life, so she hoped for an addition to her sumatriptan to more effectively treat her pain. Her optimism and internal motivation to improve gave us an opportunity to assess the efficacy of the medication over time using journaling techniques pared with multiple check-up visits.

The manuscript has internal validity with the results indicating an improvement in migraine pain with the sumatriptan/naproxen combination pill. Even in the poor-responder population, the addition of a second abortive medication with a different mechanism was likely to have a positive effect pain control, so the results make sense. The external validity of the manuscript is equally positive, as Ms. Brown showed a near immediate benefit from the combined medication. She reported that although the frequency of her migraines did not improve, she was able to abort them using the medication and was not forced to remove herself from activities, which was her goal. It was communicated that this improvement may not be permanent, and she may need to alter her regimen in the future, but she indicated that improvement in the short term was what she was most hopeful for.

\section{New Knowledge Related to Clinical Decision Science}

Ms. Brown's hesitation to completely stop sumatriptan monotherapy even though she reported it as incompletely effective represents a common trend in patient care. Patients are hesitant to start unfamiliar medications even though they are clinically recommended. In understanding her concerns, we were able to both treat her migraines with a combination pill that was similar to what she was accustomed to taking while also keeping her comfortable with the medical decision making. Generally, this should be viewed as a case study in how patient care should be modeled - ensuring patient's questions are answered with clinical reasoning backed by literature, while maintaining patient-centered decisions.

\section{Conflict Of Interest Statement}

The author declares no conflict of interest to any pharmaceutical or other entity mentioned in this manuscript.

\section{References}

1. Mathew NT, Landy S, Stark S, et al. Fixed-dose sumatriptan and naproxen in poor responders to triptans with a short half-life. Headache. 2009;49(7):971-982. https://doi.org/10.1111/i.1526-4610.2009.01458.x

2. Landy S, DeRossett SE, Rapoport A, et al. Two double-blind, multicenter, randomized, placebo-controlled, single-dose studies of sumatriptan/naproxen sodium in the acute treatment of migraine: function, productivity, and satisfaction outcomes. MedGenMed. 2007;9(2):53.

3. Edwards KR, Rosenthal BL, Farmer KU, Cady RK, Browning R. Evaluation of sumatriptan-naproxen in the treatment of acute migraine: a placebo-controlled, double-blind, cross-over study assessing cognitive function. Headache. 2013;53(4):656-664. https://doi.org/10.1111/head.12052

4. Lipton RB, Dodick DW, Adelman JU, et al. Consistency of response to sumatriptan/naproxen sodium in a placebo-controlled, crossover study. Cephalalgia. 2009;29(8):826-836. https://doi.org/10.1111/i.1468-2982.2008.01806.x

5. Silberstein S, McDonald SA, Goldstein J, et al. Sumatriptan/naproxen sodium for the acute treatment of probable migraine without aura: a randomized study. Cephalalgia. 2014;34(4):268-279. https://doi.org/10.1177/0333102413508242

6. Ebell MH, Siwek J, Weiss BD, et al. Strength of Recommendation Taxonomy (SORT): A patient-centered approach to grading evidence in the medical literature. J Am Board Fam Pract. 2004;17(1):59-67. https://doi.org/10.3122/jabfm.17.1.59 
COHEN EM. The efficacy of a sumatriptan and naproxen combination pill in a patient with chronic migraines who discontinued triptan therapy in the past due to a self-reported poor response to sumatriptan monotherapy. Clin. Res. Prac. Oct 13 2021;7(2):eP2866.

https://doi.org/10.22237/crp/1625097900
Clinical Research in Practice The Journal of Team Hippocrates

VOL 7 ISS 2 / eP2866 / OCTOBER 13, 2021 https://doi.org/10.22237/crp/1625097900

7. Dahlöf CG. Infrequent or non-response to oral sumatriptan does not predict response to other triptans--review of four trials. Cephalalgia. 2006;26(2):98-106. https://doi.org/10.1111/i.1468-2982.2005.01010.x 